Article

\title{
In Vivo Immunotoxicity of SiO2@(Y0.5Gd0.45Eu0.05)2O3 as Dual-Modality Nanoprobes
}

\author{
Xiumei Tian, Ermao Li, Fanwen Yang, Ye Peng, Jixiang Zhu, Fupo He and Xiaoming Chen * \\ Department of Biomedical Engineering, Guangzhou Medical University, Guangzhou 510182, China; \\ E-Mails: tianxiumei224@gmail.com (X.T.); bailang74624@gmail.com (E.L.); \\ yangfanwen42@gmail.com (F.Y.); pengyejlu00@gmail.com (Y.P.); zhujixiang888@gmail.com (J.Z.); \\ wild.4145@gmail.com (F.H.)
}

* Author to whom correspondence should be addressed; E-Mail: xmchenww @gmail.com; Tel./Fax: +86-20-8134-0348.

Received: 29 April 2014; in revised form: 22 May 2014 / Accepted: 4 July 2014 /

Published: 7 August 2014

\begin{abstract}
We have successfully synthesized $\mathrm{SiO}_{2} @\left(\mathrm{Y}_{0.5} \mathrm{Gd}_{0.45} \mathrm{Eu}_{0.05}\right)_{2} \mathrm{O}_{3}$ nanocomposites as a potential dual-modality nanoprobe for molecular imaging in vitro. However, their immunotoxicity assessment in vivo remains unknown. In this article, the in vitro biocompatibility of our dual-modality nanoprobes was assayed in terms of cell viability and apoptosis. In vivo immunotoxicity was investigated by monitoring the generation of reactive oxygen species (ROS), cluster of differentiation (CD) markers and cytokines in Balb/c mice. The data show that the in vitro biocompatibility was satisfactory. In addition, the immunotoxicity data revealed there are no significant changes in the expression levels of $\mathrm{CD} 11 \mathrm{~b}$ and CD71 between the nanoprobe group and the $\mathrm{Gd}$ in a diethylenetriaminepentaacetic acid (DTPA) chelator (Gd-DTPA) group $24 \mathrm{~h}$ after injection in Balb/c mice $(p>0.05)$. Importantly, there are significant differences in the expression levels of CD206 and CD25 as well as the secretion of IL-4 and the generation of ROS $24 \mathrm{~h}$ after injection $(p<0.05)$. Transmission electron microscopy (TEM) images showed that few nanoprobes were localized in the phagosomes of liver and lung. In conclusion, the toxic effects of our nanoprobes may mainly result from the aggregation of particles in phagosomes. This accumulation may damage the microstructure of the cells and generate oxidative stress reactions that further stimulate the immune response. Therefore, it is important to evaluate the in vivo immunotoxicity of these rare earth-based biomaterials at the molecular level before molecular imaging in vivo.
\end{abstract}


Keywords: immunotoxicity; reactive oxygen species; nanoprobe; gadolinium; europium; molecular imaging

\section{Introduction}

Rare earth-based nano-materials have attracted considerable attention due to their specific physicochemical characteristics in molecular imaging and medical diagnosis [1-3]. They also may do potential harm to human health because of their high surface-to-volume ratios, small size, different shapes, positive surface charges, etc. Thus, risk assessment of these materials is a particularly important scientific issue [4,5]. While toxicity assessments of rare earth-based nanomaterials are common in vitro, in vivo toxicity data remains scarce $[4,6,7]$.

As a highly reactive chemical, reactive oxygen species (ROS) are widely related to physiological and pathological phenomena. Overproduction of ROS is an important mechanism in nanotoxicity [8,9]. ROS is a critical regulator of immunity, and the expression of cluster of differentiation (CD) markers correlates to activated or proliferated immune cells. Cytokines also play a crucial role in modulating the immune response. Besides cellular oxidative stress data, the generation of ROS in vivo has not been studied extensively especially for rare earth-based nanomaterials. ROS are important regulators and suppressors of immune response [10,11]. The immune system protects the host from foreign substances including nano-based biomaterials. It is crucial to measure ROS and immunotoxicity in vivo to understand the relationship between the properties of rare earth-based nanomaterials and toxicity. Unfortunately, there are few available studies on the immunotoxicity of nano-based biomaterials in vivo $[6,12]$.

In our previous research [13], we have successfully synthesized $\mathrm{SiO}_{2} @\left(\mathrm{Y}_{0.5} \mathrm{Gd}_{0.45} \mathrm{Eu}_{0.05}\right)_{2} \mathrm{O}_{3}$ nanocomposites as a potential dual-modality nanoprobe for magnetic resonance (MR) and optical imaging in vitro, but have yet to perform systematic assessments of its immunotoxicity in vivo. In this study, we systematically evaluate the toxicity of our dual-modality nanoprobes including cell viability and apoptosis in vitro as well as the generation of ROS in peripheral blood neutrophils. We further characterize the cluster of differentiation (CD) markers in peripheral blood as well as representative cytokines in the serum of Balb/c mice. These in vivo studies are important to assess the risk assessment of these dual-modality nanoprobes before clinical use.

\section{Results and Discussion}

\subsection{Characterization of the Dual-Modality Nanoprobes}

The microstructures and morphologies of the dual-modality nanoprobes were characterized by transmission electron microscopy (TEM) and scanning electron microscope (SEM). The typical TEM and SEM images (Figure 1a-c) show spherical nanoparticles with core-shell morphologies. The samples had good dispersion and uniformity. Furthermore, the in vitro $T_{1}$-weighted MR images demonstrated that the signal intensity of the dual-modality nanoprobes enhanced with increasing $\mathrm{Gd}^{3+}$ concentration and the relaxivity $\left(r_{1}\right)$ is $4.3 \mathrm{mM}^{-1} \cdot \mathrm{s}^{-1}$ (Figure 1d,e). This suggests that our dual-modality 
nanoprobes are suitable for $T_{1}$-weighted contrast agents in vitro. Additional characterization has been reported previously [13]. In addition, zeta potential of the dual-modality nanoprobes was $(-7.31 \pm 1.45) \mathrm{mV}$ (Figure S1). This result suggested that it was difficult for the nanoprobes to combine with most proteins due to negative charge in the blood. To some extent, it is good for nanoprobes to decrease the potential toxicity.

Figure 1. Characterization of the dual-modality nanoprobes. (a,b) transmission electron microscopy (TEM) micrograph and typical histograms for the dual-modality nanoprobes and SEM (c); (d) $T_{1}$-weighted phantom MR image of the nanoprobes with $\mathrm{Gd}^{3+}$ concentrations of $0.025,0.05,0.1,0.2$ and $0.4 \mathrm{mM}$ at $1.5 \mathrm{~T}$; and (e) The relaxivity $\left(r_{1}\right)$ of the dual-modality nanoprobes by nuclear magnetic relaxation dispersion (NMRD) was at $1.5 \mathrm{~T}$.

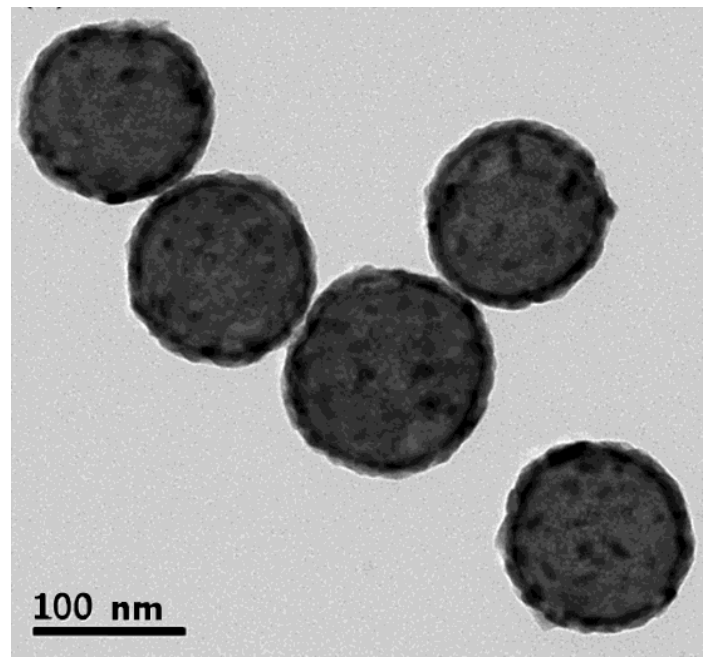

(a)

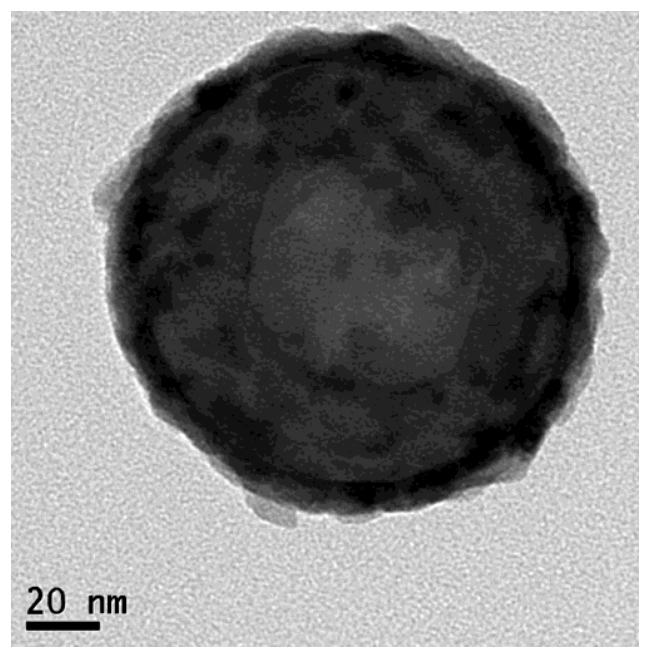

(b)

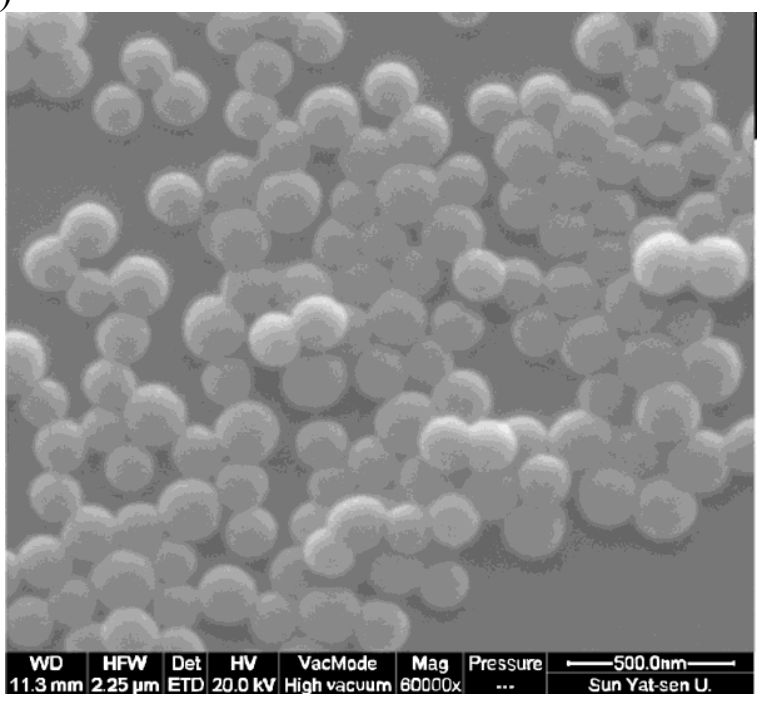

(c)

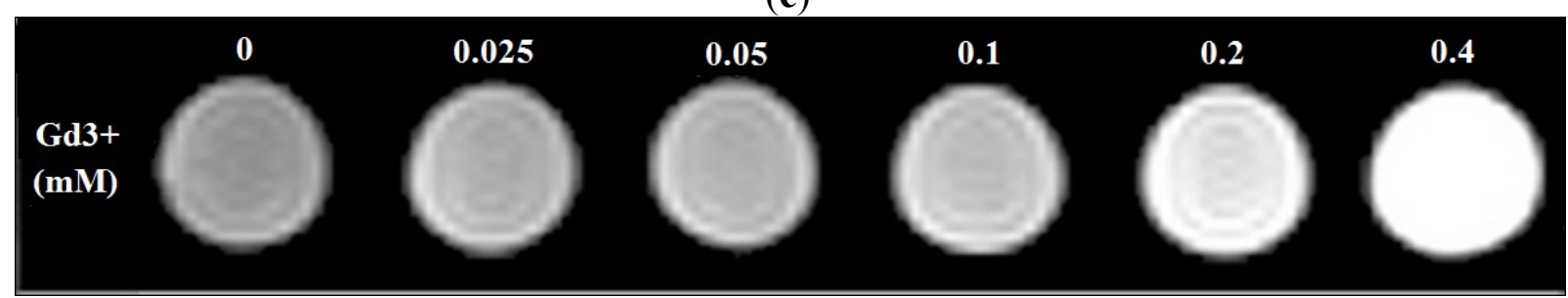

(d) 
Figure 1. Cont.

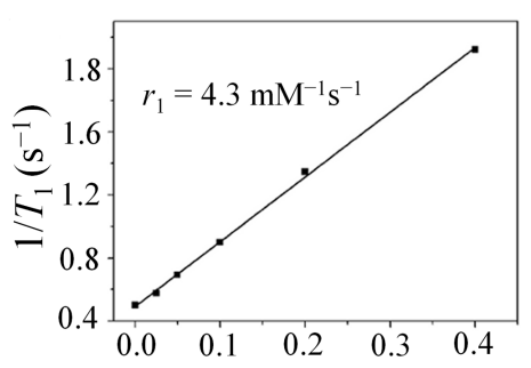

(e)

\subsection{Toxicity Effects on Macrophages in Vitro}

In general, nano-based biomaterials are mainly removed by macrophages [14], and the macrophages are more sensitive to nanoparticle exposure than others cells [15]. TEM of the murine macrophage line Raw264.7 cells were used to evaluate cell uptake. As shown in Figure 2a, few nanoprobes were absorbed by Raw 264.7 cells at $12 \mathrm{~h}$ after incubation with dual-modality nanoprobes $(100 \mu \mathrm{M} / \mathrm{L})$ and localized in phagosome. The size, zeta potential and coating of nanoprobe play significant role to affect on its cellular uptake. In addition, the viability of murine macrophage cell line Raw264.7 was evaluated by the MTT assay. Figure $2 b$ shows that the cytotoxicity of Raw264.7 cells had no significant difference after incubation for 24 and $48 \mathrm{~h}(p>0.05)$. This complements cytotoxicity data for L929 and lymphocyte cells from our precious study [13]. All cytotoxicity of the nanoprobes was negligible and indicated that the concentration of free $\mathrm{Gd}^{3+}$ ions was low.

Figure 2. The in vitro biocompatibility of the dual-modality nanoprobes. (a) Cell uptake of dual-modality nanoprobes and TEM images of Raw 264.7 cells at 12 h; (b) Cell viability of the murine macrophage line Raw 264.7 cells incubated with different concentrations ( 0.1 and $1 \mathrm{mM}$ ) of the dual-modality nanoprobes at 24 and $48 \mathrm{~h}$; and (c) Apoptosis rate of Raw264.7 cells were measured by flow cytometry $48 \mathrm{~h}$ after incubation of PBS, Gd-DTPA $(1 \mathrm{mM})$ or the dual-modality nanoprobes $(0.1$ and $1 \mathrm{mM})$. Samples were stained by Annexin V and Propidium Iodide (PI).

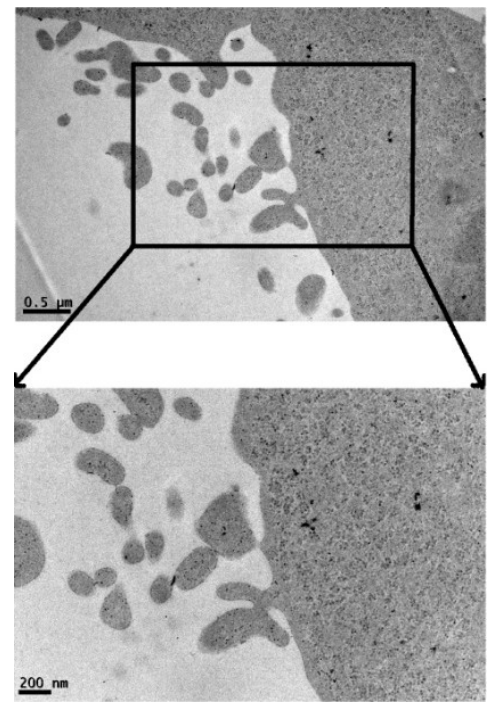

(a)

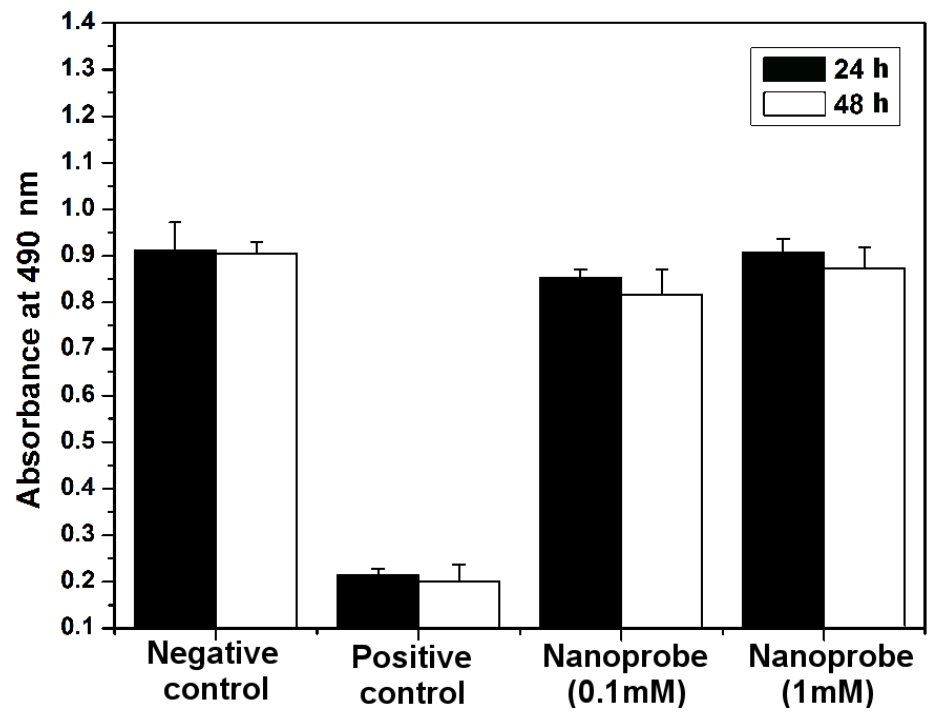

(b) 
Figure 2. Cont.

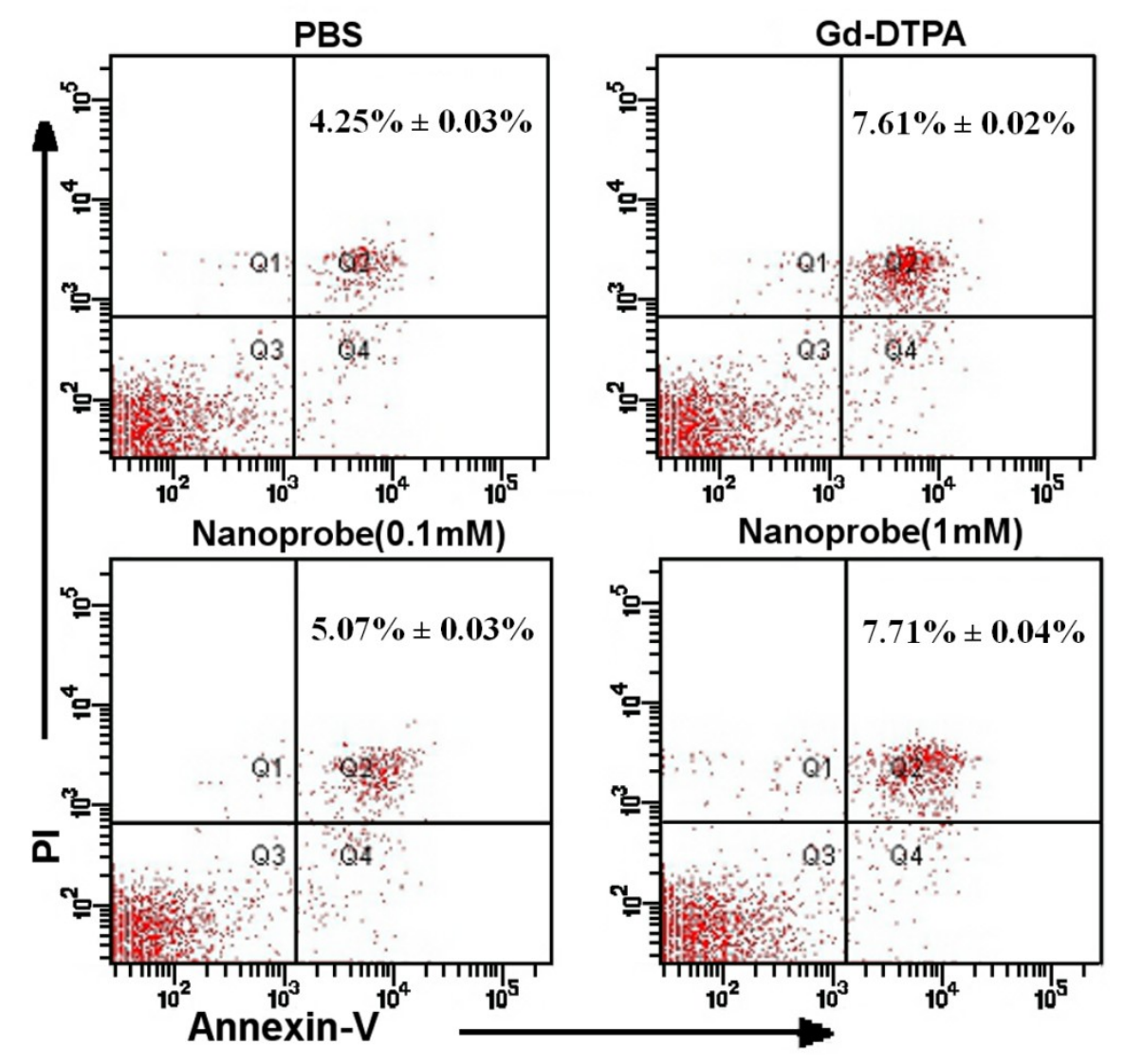

(c)

Apoptosis relates to risk assessment in vitro [16]. To further analyze the cytotoxicity, we detected the apoptosis of Raw264.7 cells after incubating the dual-modality nanoprobes for $48 \mathrm{~h}$. Figure 2c showed that no significant differences were observed between the dual-modality nanoprobe group and the Gd-DTPA group $(p>0.05)$. The results indicated that our dual-modality nanoprobes had no detrimental effect on macrophages survival. The in vitro risk assessments are consistent with data on the stability of the dual-modality nanoprobes (Supplementary 2.1). Consequently, the results of the toxic evaluation in vitro for the dual-modality nanoprobes were satisfactory.

\subsection{Toxic Effects on the Immune System in Mice}

\subsubsection{The Generation of Reactive Oxygen Species (ROS) as an Essential Regulator of Immunity}

Although the dual-modality nanoprobes showed promising biocompatibility in vitro, it is also important to evaluate their in vivo toxicity effects in mice because the culture conditions may not accurately recapitulate the in vivo immune system. Neutrophils in the peripheral blood are an important effector of host immunity. They provide first-line defense against infections [17]. Moreover, neutrophils in healthy individuals can produce up to $95 \%$ of circulating myeloperoxidase (MPO) that catalyzes the generation of ROS [18]. As a key regulator of immunity, ROS can trigger either immunosuppression or the eradication of pathogens during tissue-restoration [11]. Furthermore, we investigated the generation of ROS in neutrophils of the peripheral blood in Balb/c mice after 
injection. Figure 3 showed that the generation of ROS in peripheral blood neutrophils on the dual-modality nanoprobes group was 2-fold increased comparing to the Gd-DTPA group at 1 day. However, no significant differences were seen between the negative group and the dual-modality nanoprobe group after 7 days. The in vivo data showed that the dual-modality nanoprobes stimulated the generation of ROS at 1 day, and cells or tissues were affected by the oxidative stress to some degree. However, the level of ROS returned to the baseline at 7 days by physiological regulation. In many settings, ROS is relative to the host innate and adaptive immune response $[19,20]$. Therefore, we further studied the expression levels of certain CD markers and cytokines in the immune response at 1 day.

Figure 3. The generation of ROS in peripheral blood neutrophils was measured by flow cytometry 1 and 7 days after injection in Balb/c mice $(20 \mu \mathrm{mol} / \mathrm{kg}$, mean $\pm \mathrm{SD}, n=5)$.

$* p<0.05$ compared with the Gd-DTPA group.

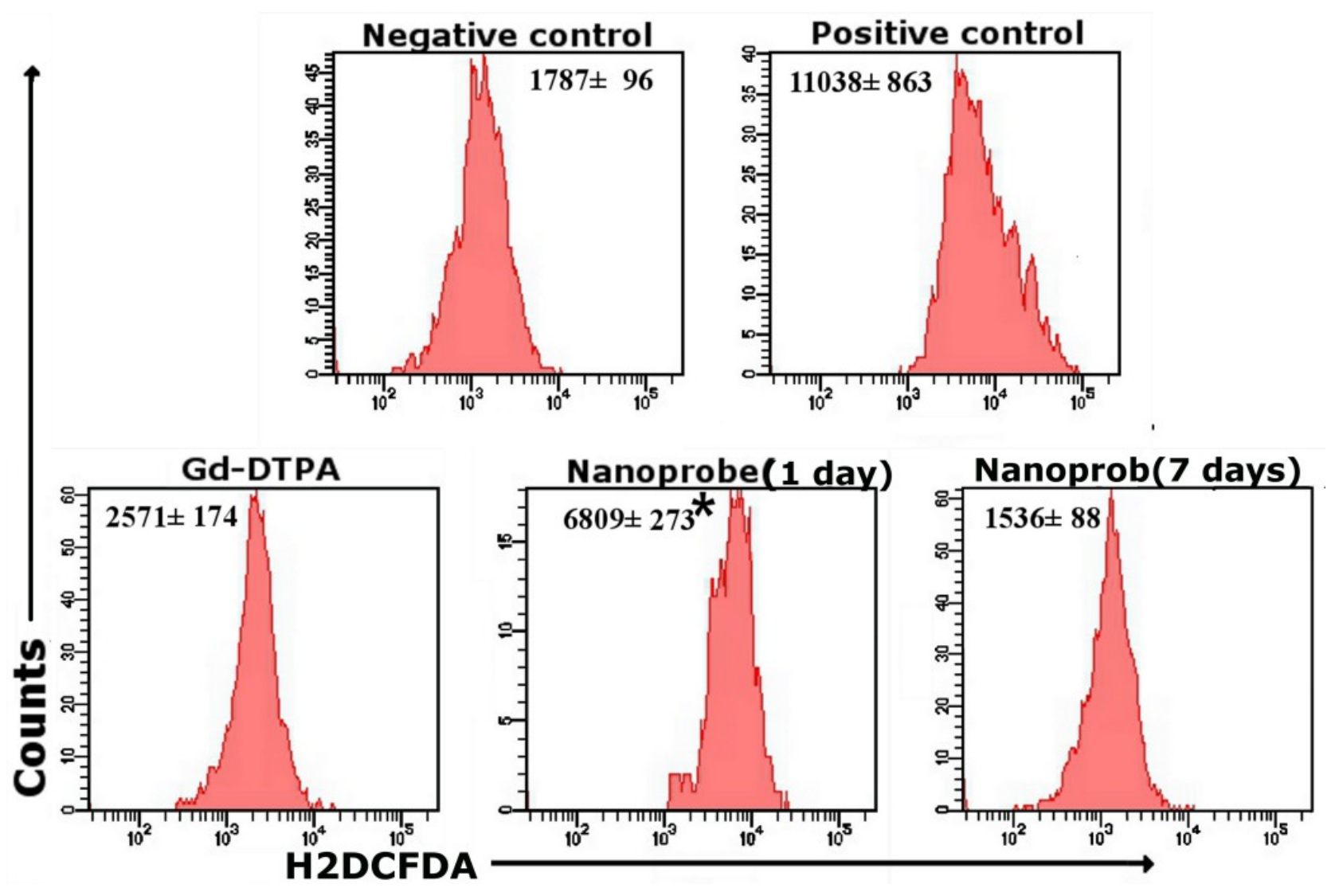

\subsubsection{The Expression of Representative CDs on the Immune Response}

CD206 is expressed on most tissue macrophages [21]; CD11b is mainly present on blood monocytes and immature macrophages [22]. CD25 and CD71 are surface markers in activated T cells [23]. Therefore, their expression levels are an important parameter in evaluating monocytes/macrophages and activated $\mathrm{T}$ cells. We investigated expression levels of CD markers from monocytes/macrophages in peripheral blood. The expression levels of CD206 and CD25 were significantly increased from the nanoprobe group compared to the Gd-DTPA group (Figures 4a and S2). The number of macrophages and activated $\mathrm{T}$ cells was elevated. Importantly, expression levels of all the $\mathrm{CD}$ markers from the 
nanoprobe group are elevated versus the negative control group. The results indicated that our dual-modality nanoprobes might stimulate the immune cells via the over-generation of ROS after injection.

Figure 4. Toxicity of the immune response measured by flow cytometry $24 \mathrm{~h}$ after injection in Balb/c mice $(20 \mu \mathrm{mol} / \mathrm{kg}$, mean $\pm \mathrm{SD} ; n=5)$. (a) The expression levels of CD206, CD11b, CD71 and CD25 in peripheral blood; and (b) The levels of IL-1 $\beta$, IL-2 and IL-4 in serum. * $p<0.05$ compared with the Gd-DTPA group.

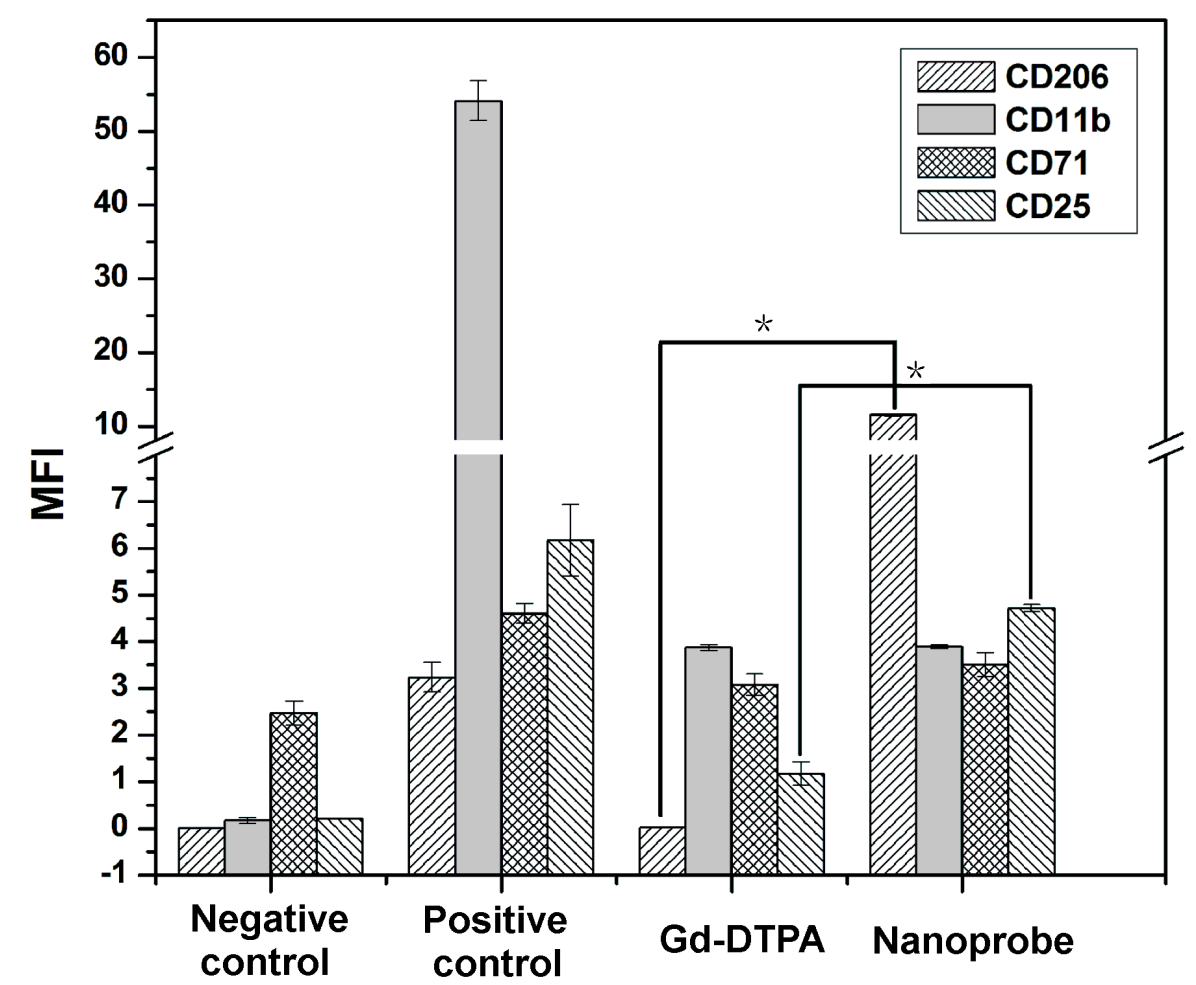

(a)

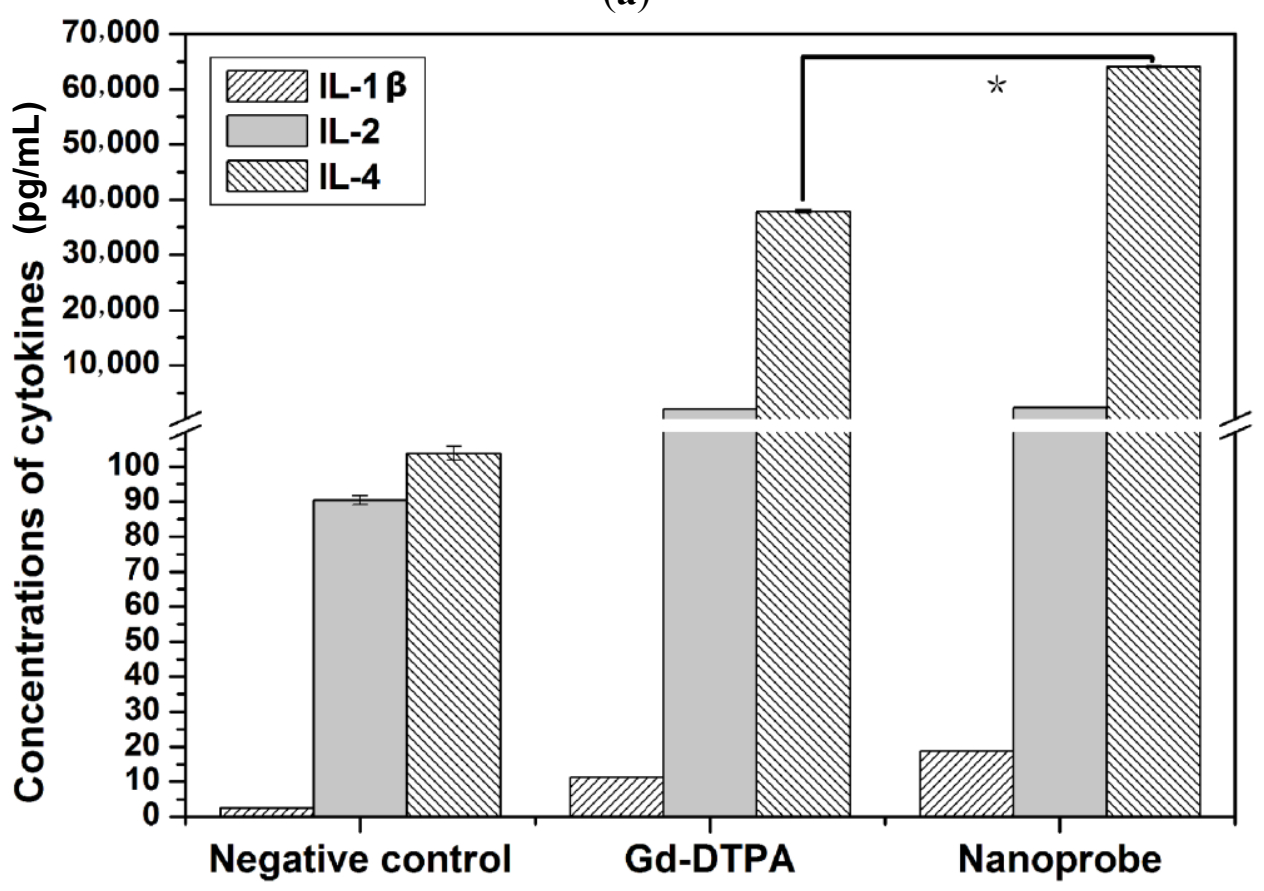

(b) 


\subsubsection{The Secretion of Representative Cytokines on the Immune Response}

To further elaborate on the in vivo immunotoxicity, we performed experiments on the secretion of representative cytokines. Because cytokines play an important role in modulating the host immune system, it is important to investigate how their expression might change in mice [24]. Interleukin-1 $\beta$ (IL-1 $\beta$ ) is an endogenous pyrogen and is involved in the inflammatory response. The IL-1 $\beta$ stimulates thymocyte proliferation by inducing IL-2 release [25]. Interleukin-2 (IL-2) is a T-cell growth factor, and a required protein for T-cell proliferation and other regulators of the immune response [26]. Interleukin-4 (IL-4) has many biological roles including the stimulation of T-cell proliferation and B-cell activation [27].

The levels of these cytokines are presented in Figure $4 \mathrm{~b}$. We observed that there is a significant difference in the secretion of IL-4 between the nanoprobe group and the the Gd in a diethylenetriaminepentaacetic acid (DTPA) chelator (Gd-DTPA) group $(p<0.05)$. Moreover, the secretion of IL-2 and IL-4 from the nanoprobe group was increased versus the negative control group. The results indicated that some of the T-cells or B-cells were activated by the nanoprobes in mice, and T-cells might be stimulated to proliferation. But the immunotoxicity cannot be regulated endogenously. Consequently, it is important to evaluate immunotoxicity in vivo when studying the risk assessment of the rare earth-based nanomaterials.

\subsection{Biodistribution of the Dual-Modality Nanoprobes at the Subcellular Level}

The biodistribution of nano-based biomedicine relates to their biocompatibility [28]. To further visualize the biodistribution of nanoprobes, we used TEM. Sections of the liver and lung in mice were observed $24 \mathrm{~h}$ after injection (Figure 5a). Few nanoprobes were located in the cytoplasm of epithelial cells in the liver and lung. The results show that our nanoprobes were dispersed inside the phagosomes of the tissues and exhibited aggregation, which is consistent with the other researches [29,30]. As such, the dual-modality nanoprobes did slight damage to the microstructure or ultrastructure of the cells, and this may induce some of the toxic effects in vivo.

\subsection{Pathological Analysis of the Dual-Modality Nanoprobes}

The pathological biopsy assay (Leica DMIRBE, Wetzlar, Germany) was conducted on major organs including brain, heart, liver, kidney, spleen and lung $24 \mathrm{~h}$ after injection. Samples were stained with H\&E and prussian blue. As shown in Figures 5b and S3, there were no abnormal changes in any histological sections. This kind of the pathological examination provides data on the gross pathologic evaluation. The histologic and microscopic examinations demonstrated that the nanoprobes had not any influence on the cellular integrity and tissue morphology. Therefore, it is inevitable for in vivo immunotoxicity assessment to evaluate the potential health risks on the rare earth-based biomaterials. 
Figure 5. (a) The biodistribution of the dual-modality nanoprobes at the subcellular level. transmission electron microscopy (TEM) images from the liver and lung in mice $24 \mathrm{~h}$ after intravenous administration; and (b) Pathological biopsy assay on brain, heart, liver and kidney on the dual-modality nanoprobes stained with hematoxylin and eosin (H\&E) and prussian blue.

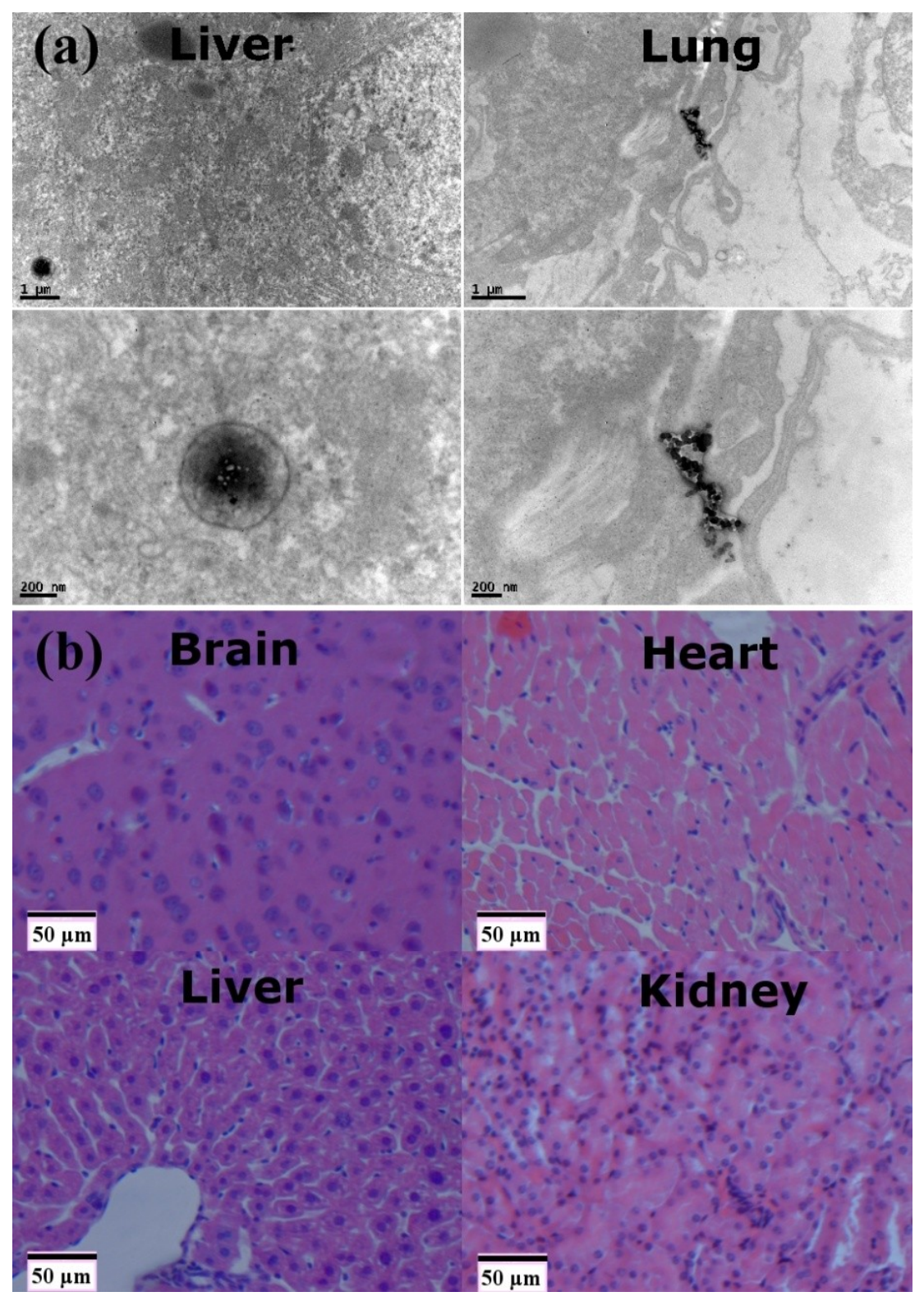

\section{Experimental Section}

\subsection{Synthesis and Characterization of the Dual-Modality Nanoprobes}

Dual-modality nanoprobes, $\mathrm{SiO}_{2} @\left(\mathrm{Y}_{0.5} \mathrm{Gd}_{0.45} \mathrm{Eu}_{0.05}\right)_{2} \mathrm{O}_{3}$ nanocomposites, were prepared according to our previous report [13]. The characterization mainly used TEM (A JEM-2010 HR, JEOL Ltd., Tokyo, Japan) and scanning electronic microscopy (SEM) (LEO-1530VP, LEO, Oberkochen, Germany). The T1-weighted MRI and the relaxivity $\left(r_{1}\right)$ were measured by a $1.5 \mathrm{~T}$ MRI scanner (General Electric, Sigma, Milwaukee, WI, USA). 


\subsection{The in Vitro Biocompatibility of the Dual-Modality Nanoprobes}

\subsubsection{Cytotoxicity Assay}

Murine macrophage RAW264.7 cells were cultured with Dulbecco's Modified Eagle Media (DMEM, Gibco, Grand Island, NY, USA) and 10\% fatal bovine serum (FBS, Gibco, Grand Island, NY, USA) in 96-well plates and incubated with different concentrations of nanoprobes (0.1 and $1 \mathrm{mM})$ during the logarithmic growth period for 24 and $48 \mathrm{~h}$. Treatment with culture media and $0.5 \%$ dimethyl sulfoxide (DMSO, Sigma-Aldrich Corp., St. Louis, MO, USA) served as the negative control and the positive control, respectively. DMEM was removed carefully $44 \mathrm{~h}$ after incubation followed by $20 \mu \mathrm{L}$ of 3-(4,5-dimethylthiazol-2-yl)-2,5-diphenyltetrazolium bromide (MTT, Sigma-Aldrich Corp., St. Louis, MO, USA) and incubated for $4 \mathrm{~h}$ followed by $100 \mu \mathrm{L}$ dimethyl sulfoxide (DMSO) for $10 \mathrm{~min}$ with gentle shaking. Absorbance was measured at $490 \mathrm{~nm}$ using a microplate reader (Bio-Rad, San Diego, CA, USA).

\subsubsection{Apoptosis Assay}

The murine macrophage RAW264.7 cells were incubated with DMEM and 10\% FBS in 6-well plates and treated with PBS (phosphate-buffered saline; negative control), LPS (lipopolysaccharide; positive control, Sigma-Aldrich Corp., St. Louis, MO, USA), the Gd in a diethylenetriaminepentaacetic acid (DTPA) chelator (Gd-DTPA) (commercial MRI contrast agent; $1 \mathrm{mM}$, Magnevist, Berlex Laboratories, Wayne, NJ, USA) and dual-modality nanoprobes $(0.1$ and $1 \mathrm{mM})$ for $48 \mathrm{~h}$. We then removed the culture media and digested the cells with $0.5 \%$ trypsin- $0.2 \%$ EDTA (Ethylene diamine tetraacetic acid) (Sigma-Aldrich Corp., St. Louis, MO, USA) for $3 \mathrm{~min}$. Cells were washed twice with cold PBS by gentle shaking. Samples were stained with Annexin-V/PI (propidium iodide) (kit from eBioscience, San Diego, CA, USA). We resuspended the cells in $200 \mu \mathrm{L}$ binding buffer $(1 \times)$ at $1 \times 10^{6}$ cells $/ \mathrm{mL}$ and then added $5 \mu \mathrm{L}$ Annexin V-fluorescein isothiocyanate isomer (FITC) into $195 \mu \mathrm{L}$ of the cell suspension. This was mixed and incubated for $10 \mathrm{~min}$ at room temperature. Cells were washed twice with $200 \mu \mathrm{L}$ binding buffer $(1 \times)$ and resuspended in $190 \mu \mathrm{L}$ binding buffer $(1 \times)$. We then added $10 \mu \mathrm{L}$ propidium iodide (PI) $(20 \mu \mathrm{g} / \mathrm{mL})$ into the cells. Finally, the samples were measured with a FACScan (Becton Dickinson, Mountain View, CA, USA).

\subsection{In Vivo Immunotoxicity of the Dual-Modality Nanoprobes}

Twenty male Balb/c mice were divided into four groups at random and injected via tail vein: (a) PBS (100 $\mu \mathrm{L}$; negative control); (b) LPS (5 mg/kg); (c) Gd-DTPA (20 $\mu \mathrm{mol} / \mathrm{kg}$ ); and (d) the dual-modality nanoprobes $(20 \mu \mathrm{mol} / \mathrm{kg})$. Samples were obtained at 1 day after injection and measured by flow cytometry (Becton Dickinson, Mountain View, CA, USA).

\subsubsection{ROS Assay on Peripheral Blood Neutrophils}

Peripheral blood from the tail vein $(20 \mu \mathrm{L})$ was added to a tube with heparin sodium $(4 \mu \mathrm{L})$. Erythrocytes were lysed by red blood cell lysis buffer $(1 \mathrm{~mL}$, Beyotime, Haimen, China) in the dark for $2 \mathrm{~min}$. After removing the supernatant, PBS $(2 \mathrm{~mL})$ was added and the sample was centrifuged at 
$500 \times g$ for 5 min at $4{ }^{\circ} \mathrm{C}$. We next removed the supernatant. The carboxy derivative of fluorescein, carboxy-H2DCFDA (C400), (H2DCFDA, Invitrogen, California, CA, USA) was added to the samples $\left(1 \times 10^{6}\right.$ cells $)$ at a final concentration of $5 \mu \mathrm{M}$. Cells were mixed thoroughly in the dark for $20 \mathrm{~min}$ and PBS $(400 \mu \mathrm{L})$ was added.

\subsubsection{Expression Levels of Representative CD Markers in Peripheral Blood}

Twenty microliters of peripheral blood from the ophthalmic vein was collected in a tube with heparin sodium $(4 \mu \mathrm{L})$. The erythrocytes were lysed by red blood cell lysis buffer $(1 \mathrm{~mL}$, Beyotime, Haimen, China) in the dark for $2 \mathrm{~min}$. We then removed the supernatant. Next, $1 \times 10^{6}$ cells were stained in the dark for 30 min with anti-mouse CD206-PE, anti-mouse CD11b-PE, anti-mouse F4/80 allophycocyanion (APC), anti-mouse CD25-FITC, anti-mouse CD71-FITC and anti-mouse CD3-PE (Tianjin Sungene Biotech Co., Tianjing, China). This was followed by the addition of $2 \mathrm{~mL}$ PBS, centrifugation at $1200 \times \mathrm{g}$ for $5 \mathrm{~min}$ at room temperature, and resuspension in $400 \mu \mathrm{L}$ PBS.

\subsubsection{Levels of Cytokines in Serum}

Peripheral blood was obtained by the vena ophthalmica in $1.5 \mathrm{~mL}$ eppendorf tubes. Samples were kept at room temperature for $45 \mathrm{~min}$ and then centrifuged at $3000 \times \mathrm{g}$ for $10 \mathrm{~min}$ at $4{ }^{\circ} \mathrm{C}$. The supernatant was transferred into new tubes and IL-1 $\beta$, IL-2 and IL-4 levels were measured with ELISA kits (RayBiotech, Inc., Guangzhou, China) according to the provided instructions.

\subsubsection{Statistical Analysis}

Data are means \pm standard deviation (SD). Statistical analysis was performed by a one-way ANOVA and $t$-tests using Origin 8.0 software (OriginLab Co., Northampton, MA, USA). Data was considered significant when $p<0.05$.

\section{Conclusions}

The dual-modality nanoprobe is a contrast agent for magnetic resonance/optical imaging and has satisfactory biocompatibility in vitro (Figures 2 and S4). Unfortunately, the in vivo immunotoxicity is poor because of its nano-structure. The mechanism of immunotoxicity might be that the microstructure of the cells is slightly damaged by the overproduction of reactive oxygen species (ROS), which might stimulate the immune response in mice after injection. Therefore, there is no reason to study the nanoprobes with magnetic resonance imaging (MRI) in vivo, especially in light of the time and cost associated with MRI. This also suggests no need for clinical translation. It is very important for rare earth-based nanomaterials to be evaluated for immunotoxicity in vivo. This work suggests tools for evaluation of immunotoxicity in vivo.

\section{Acknowledgments}

This work was funded by the Postdoctoral Science Foundation of China (No. 2014M552182), and the National Natural Science Foundation of China (No. 81071207, 81271622). 


\section{Author Contributions}

X.T. and X.C. conceived and designed the experiments; E.L. analyzed the data; F.Y. and Y.P. contributed reagents/materials/analysis tools; X.T., J.Z. and F.H. wrote the manuscript; X.C. and X.T. revise the manuscript.

\section{Conflicts of Interest}

The authors declare no conflict of interest.

\section{References}

1. Mayer, F.; Zhang, W.; Brichart, T.; Tillement, O.; Bonnet, C.S.; Toth, E.; Peters, J.A.; Djanashvili, K. Nanozeolite-LTL with $\mathrm{Gd}^{\mathrm{III}}$ deposited in the large and $\mathrm{Eu}^{\mathrm{III}}$ in the small cavities as a magnetic resonance optical imaging probe. Chemistry 2014, 20, 3358-3364.

2. Chen, B.; Zhang, H.; Du, N.; Zhang, B.; Wu, Y.; Shi, D.; Yang, D. Magnetic-fluorescent nanohybrids of carbon nanotubes coated with $\mathrm{Eu}, \mathrm{Gd}$ co-doped $\mathrm{LaF}_{3}$ as a multimodal imaging probe. J. Colloid Interface Sci. 2012, 367, 61-66.

3. Yang, D.; Zhao, Y.; Guo, H.; Li, Y.; Tewary, P.; Xing, G.; Hou, W.; Oppenheim, J.J.; Zhang, N. $\left[\mathrm{Gd} @ \mathrm{C}_{82}(\mathrm{OH})_{22}\right]_{n}$ nanoparticles induce dendritic cell maturation and activate Th1 immune responses. Am. Chem. Soc. Nano 2010, 4, 1178-1186.

4. Fischer, H.C.; Chan, W.C. Nanotoxicity: The growing need for in vivo study. Curr. Opin. Biotechnol. 2007, 18, 565-571.

5. Donner, M.; Tran, L.; Muller, J.; Vrijhof, H. Genotoxicity of engineered nanomaterials. Nanotoxicology 2010, 4, 345-346.

6. Dobrovolskaia, M.A.; Germolec, D.R.; Weaver, J.L. Evaluation of nanoparticle immunotoxicity. Nat. Nanotechnol. 2009, 4, 411-414.

7. Maccormack, T.J.; Clark, R.J.; Dang, M.K.; Ma, G.; Kelly, J.A.; Veinot, J.G.; Gross, G.G. Inhibition of enzyme activity by nanomaterials: Potential mechanisms and implications for nanotoxicity testing. Nanotoxicology 2012, 6, 514-525.

8. Sophie, L.; Jorge, B. Biomedical applications and potential health risks of nanomaterials: Molecular mechanisms. Curr. Mol. Med. 2006, 6, 651-663.

9. Bondarenko, O.; Juganson, K.; Ivask, A.; Kasemets, K.; Mortimer, M.; Kahru, A. Toxicity of Ag, $\mathrm{CuO}$ and $\mathrm{ZnO}$ nanoparticles to selected environmentally relevant test organisms and mammalian cells in vitro: A critical review. Arch. Toxicol. 2013, 87, 1181-1200.

10. Vandebriel, R.J.; de Jong, W.H. A review of mammalian toxicity of $\mathrm{ZnO}$ nanoparticles. Nanotechnol. Sci. Appl. 2012, 5, 61-71.

11. Yang, Y.; Bazhin, A.V.; Werner, J.; Karakhanova, S. Reactive oxygen species in the immune system. Int. Rev. Immunol. 2013, 32, 249-270.

12. Kermanizadeh, A.; Vranic, S.; Boland, S.; Moreau, K.; Baeza-Squiban, A.; Gaiser, B.K.; Andrzejczuk, L.A.; Stone, V. An in vitro assessment of panel of engineered nanomaterials using a human renal cell line: Cytotoxicity, pro-inflammatory response, oxidative stress and genotoxicity. BMC Nephrol. 2013, 14, 96. 
13. Liu, H.; Li, L.; Tian, X.-M.; Hu, W.-Y.; Kuang, X.-Y.; Shao, Y.-Z. Synthesis and characterization of bifunctional $\mathrm{SiO}_{2} @\left(\mathrm{Y}_{0.95-x} \mathrm{Gd}_{x} \mathrm{Eu}_{0.05}\right)_{2} \mathrm{O}_{3}$ nanocomposites for magnetic resonance and optical imaging. Eur. J. Inorg. Chem. 2012, 34, 5677-5684.

14. Gref, R.; Minamitake, Y.; Peracchia, M.T.; Trubetskoy, V.; Torchilin, V.; Langer, R. Biodegradable long-circulating polymeric nanospheres. Science 1994, 263, 1600-1603.

15. Nguyen, K.C.; Seligy, V.L.; Tayabali, A.F. Cadmium telluride quantum dot nanoparticle cytotoxicity and effects on model immune responses to Pseudomonas aeruginosa. Nanotoxicology 2013, 7, 202-211.

16. Tamura, T.; Ishihara, M.; Lamphier, M.S.; Tanaka, N.; Oishi, I.; Aizawa, S.; Matsuyama, T.; Mak, T.W.; Taki, S.; Taniguchi, T. An IRF-1-dependent pathway of DNA damage-induced apoptosis in mitogen-activated T lymphocytes. Nature. 1995, 376, 596-599.

17. Hao, S.; Andersen, M.; Yu, H. Detection of immune suppressive neutrophils in peripheral blood samples of cancer patients. Am. J. Blood Res. 2013, 3, 239-245.

18. Cao, L.Y.; Soler, D.C.; Debanne, S.M.; Grozdev, I.; Rodriguez, M.E.; Feig, R.L.; Carman, T.L.; Gilkeson, R.C.; Orringer, C.E.; Kern, E.F.; et al. Psoriasis and cardiovascular risk factors: increased serum myeloperoxidase and corresponding immunocellular overexpression by $\mathrm{Cd}_{11} \mathrm{~b}^{+}$ CD68 ${ }^{+}$macrophages in skin lesions. Am. J. Transl. Res. 2013, 6, 16-27.

19. Gaddis, D.E.; Michalek, S.M.; Katz, J. Requirement of TLR4 and CD14 in dendritic cell activation by Hemagglutinin B from Porphyromonas gingivalis. Mol. Immunol. 2009, 46, 2493-2504.

20. Kim, H.W.; Cho, S.I.; Bae, S.; Kim, H.; Kim, Y.; Hwang, Y.I.; Kang, J.S.; Lee, W.J. Vitamin C up-regulates expression of CD80, CD86 and MHC class II on dendritic cell line, DC-1 via the activation of p38 MAPK. Immune Netw. 2012, 12, 277-283.

21. Akbarshahi, H.; Menzel, M.; Posaric, B.M.; Rosendahl, A.; Andersson, R. Enrichment of murine $\mathrm{CD} 8^{+} \mathrm{CCR}^{+}$and $\mathrm{CD} 8^{+} \mathrm{CD} 206^{+}$lung macrophages in acute pancreatitis-associated acute lung injury. PLoS One. 2012, 7, e42654.

22. Roca, H.; Varsos, Z.S.; Sud, S.; Craig, M.J.; Ying, C.; Pienta, K.J. CCL2 and interleukin-6 promote survival of human $\mathrm{CD}_{1} 1 \mathrm{~b}^{+}$peripheral blood mononuclear cells and induce M2-type macrophage polarization. J. Biol. Chem. 2009, 284, 34342-34354.

23. Samarasinghe, S.; Mancao, C.; Pule, M.; Nawroly, N.; Karlsson, H.; Brewin, J.; Openshaw, P.; Gaspar, H.B.; Veys, P.; Amrolia, P.J. Functional characterization of alloreactive T cells identifies CD25 and CD71 as optimal targets for a clinically applicable allodepletion strategy. Blood 2010, 115, 396-407.

24. Woolfson, A.; Ellmark, P.; Chrisp, J.S.; Scott, M.A.; Christopherson, R.I. The application of CD antigen proteomics to pharmacogenomics. Pharmacogenomics 2006, 7, 759-771.

25. Mattuzzi, S.; Barbi, S.; Carletto, A.; Ravagnani, V.; Moore, P.S.; Bambara L.M.; Scarpa, A. Association of polymorphisms in the $I L I B$ and $I L 2$ genes with susceptibility and severity of systemic sclerosis. J. Rheumatol. 2007, 34, 997-1004.

26. Smith, K.A. Interleukin-2: Inception, impact, and implications. Science 1988, 240, 1169-1176.

27. Charteris, D.G.; Lightman, S.L. Comparison of the expression of interferon gamma, IL2, IL4, and lymphotoxin mRNA in experimental autoimmune uveoretinitis. Br. J. Ophthalmol. 1994, 78, 786-790. 
28. Dobrovolskaia, M.A.; Aggarwal, P.; Hall, J.B.; Mcneil, S.E. Interaction with the immune system and its potential effects on nanoparticle biodistribution. Mol. Pharm. 2008, 5, 487-495.

29. Van Schooneveld, M,M.; Vucic, E.; Koole, R.; Zhou, Y.; Stocks, J.; Cormode, D.P.; Tang, C.Y.; Gordon, R.E.; Nicolay, K.; Meijerink, A.; et al. Improved biocompatibility and pharmacokinetics of silica nanoparticles by means of a lipid coating: A multimodality investigation. Nano Lett. 2008, 8, 2517e25.

30. Shao, Y.; Tian, X.; Hu, W.; Zhang Y.; Liu, H.; He, H.; Shen, Y.; Xie, F.; Li, L. The properties of $\mathrm{Gd}_{2} \mathrm{O}_{3}$-assembled silica nanocomposite targeted nanoprobes and their application in MRI. Biomaterials 2012, 33, 6438-6446.

(C) 2014 by the authors; licensee MDPI, Basel, Switzerland. This article is an open access article distributed under the terms and conditions of the Creative Commons Attribution license (http://creativecommons.org/licenses/by/3.0/). 